\title{
T-Wave Changes Due to Cardiac Deformation Are Dependent on the Temporal Relationship Between Repolarization and Diastolic Phase
}

\author{
Robin Moss, Eike M Wülfers, Gunnar Seemann \\ Institute for Experimental Cardiovascular Medicine, \\ University Heart Center Freiburg · Bad Krozingen, Medical Center - University of Freiburg, \\ and Faculty of Medicine, University of Freiburg, Freiburg, Germany
}

\begin{abstract}
Over the last years computational modelling of the ECG has seen a rise in popularity. In general, the ECG is calculated based on myocyte membrane voltages and so called forward calculating of the potentials on the body surface. In nearly all cases though the deformation of the heart is neglected. This becomes a more prominent problem when looking at the T-wave. Within the ECG the T-wave shows the repolarization of the ventricles back to their resting potential. This can align with different phases of the contraction cycle, depending on action potential duration and temporal delay between electrical excitation and mechanical response.

In this study we present a finite element, fully coupled electro-mechanical model, which is deforming within a torso geometry. By artificially shifting the membrane potentials in time, we can assess how different phases of the contraction cycle affect $T$-wave properties.

In contrast to previous studies our results show little effect of deformation on T-wave amplitude, and only minor morphology changes, delaying the peak of the T-wave. By artificially shifting the potentials to a more contracted state these effects were amplified.
\end{abstract}

\section{Introduction}

Computational cardiac modelling can help identifying key aspects in the functioning of the heart. The modelling itself can be subdivided into the electrophysiological component and the mechanical component. The electrophysiological component describes all parts affiliated to action potential generation. This includes excitation propagation through the myocardial tissue as well up to later calculation of the ECG. Further, one can expand this to also include the tension generation of depolarized cells on a single cell level [1]. Based on this tension development one can then calculate the mechanical part, i.e., the resulting deformation and thus pumping of the heart. Cou- pling of the two components is crucial, as they both influence each other. In this study, we want to take a closer look at the impact the deformation itself has on the Twave of the ECG. Depending on the temporal alignment of the two components the repolarization of ventricular myocytes, responsible for the T-wave, can align with different phases of the cardiac contraction cycle. In a previous study, using manually extracted deformation from cine-MRI, it was shown that cardiac mechanical activity decreases T-wave amplitude [2], independent of time of repolarization. Yet, as the deformation was extracted manually, an over/understimation of basal as well as radial movement is possible. Further, the delay between electrical and mechanical activation can only be estimated. In contrast, a study using a small wedge of tissue showed that in a pseudo-ECG the T-wave equivilent amplitude does increase if deformation is included [3].

To overcome the limitations of the previous studies, our goal was to create a fully coupled electro-mechanical heart, which was then incorporated into a torso to study the effects of cardiac deformation on the ECG.

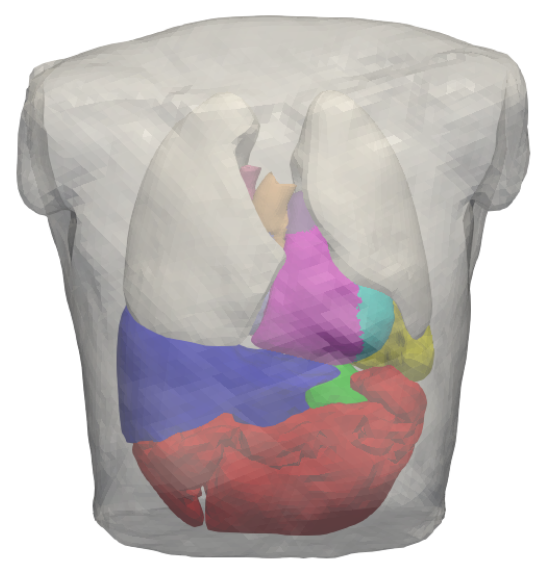

Figure 1. Geometrical model of heart and torso, based on the same volunteer as used in [1][4][12]. 


\section{Methods}

\subsection{Geometrical Modeling}

Both models, the one used for electrophysiological simulations, as well as the one used to determine the deformation, are based on data from the same volunteer [2], see Fig. 1. The electrophysiological geometry comprises $10^{6}$ and the mechanical one $84 \mathrm{k}$ elements (450k DOF). Out of these $84 \mathrm{k}$ elements, $42 \mathrm{k}$ elements describe the ventricles, $17 \mathrm{k}$ elements the atria, and the rest the surrounding tissue, aorta, pulmonary artery and pulmonary veins. The anisotropic myocyte orientation was set according to [4] for the ventricles and [5] for the atria.

\subsection{Electrophysiological Modeling}

Simulations were performed using the ventricular human myocyte cell model by TenTusscher et al. [6]. The ordinary differential equations describing cellular dynamics were solved using the forward Euler method with a time step of $10 \mu \mathrm{s}$. Excitation propagation was calculated by solving the monodomain equation discretized with first order finite elements in space, and second order CrankNicolson scheme $(\theta=0.5)$ :

$$
K\left(\theta v_{\theta}^{n+1}+(1-\theta) v_{\theta}^{n}\right)=M(\kappa+1) \beta C_{m} \frac{v_{\theta}^{n+1}-v_{\theta}^{n}}{\Delta t}
$$

with $K$ being the stiffness matrix, $M$ the mass matrix, $\kappa$ a factor between intracellular and extracellular conductance $\left(\sigma_{i}=\kappa \sigma_{e}\right), C_{m}$ the membrane capacitance, and $v_{\theta}$ the transmembrane voltage $\left(V_{m}\right)$.

Based on the concentration of $\mathrm{Ca}^{2+}$ bound to troponin, the resulting tensions developed within the single nodes is then computed according to the model by Rice et al. [1], with parameters from [3] based on human myocyte tension development. The maximum reference tension for the cell model was set to $15 \mathrm{kPa}$, to obtain a physiological left ventricular ejection fraction of approximately $55 \%$. Additionally, as needed for a concordant T-wave, and to enable a direct comparison with the previous work, an apico-basal heterogeneity in the slow potassium conductivity $g_{K, s}$ was implemented [2], similar to the one used in [7]. Further, the ratio of endocardial, mid, and epicardial cells [6], which showed the most prominent T-wave throughout the ventricular wall, was used (20\% Endo, 30\% Mid, 50\% Epi) [2].

Prior to the simulation, the electrophysiogical model was initialized by bringing the respective cell models into a steady state in a single cell environment.

\subsection{Biomechanical Modeling}

Cardiac mechanical modelling was done using CardioMechanics, which is described in [8]. In short, the kinematics are determined by the governing equation for balance of linear momentum which can be transformed into the discrete weak form of the Lagrangian formulation:

$$
\begin{aligned}
& \int_{\Omega_{0}} \rho_{0} N_{I} N_{J} d \Omega_{0} \ddot{u}_{j J}+\int_{\Omega_{0}} \frac{\delta N_{i}}{\delta X_{j}} P_{j i} d \Omega_{0}- \\
& \int_{\Omega_{0}} N_{I} \rho_{0} b_{i} d \Omega_{0}-\int_{\Gamma_{t_{i}}^{0}} N_{I} \bar{t}_{i}^{0} d \Gamma_{0}=0
\end{aligned}
$$

with $i$ and $j$ being the nodal coordinates $(x, y, z), I$ and $J$ the node indices, $\rho_{0}$ the initial mass density, $\frac{\delta N_{I}}{\delta X_{j}}$ the derivative of the shape functions, $P_{j i}$ the nominal stress, $b_{i}$ the body force, and $u$ the sought-after displacement. As for discretization, quadratic tetrahedrons were used as they are not as prone to volume locking as linear ones. Further, the tissue is assumed to be hyperelastic, anisotropic, and nearly incompressible. Material properties are described using the Guccione material law [9], with the parameters set according to [10] for the ventricles and according to [8] for the surrounding tissue, atria, and bulk modulus. The previously described single cell tension development is added to the first component of the PiolaKirchhoff stress tensor, thus inducing tension development in the long axis of the myocytes. Thereupon, the acceleration and velocity were determined using the Newmark beta scheme $(\beta=0.25, \gamma=0.5)$ and solved using the MUMPS direct solver distributed with the PETSc software framework [11], with a maximum time step of $1 \mathrm{~ms}$.

Different to [8], the apex was not fixed but the stiffness of the surrounding tissue in the apical region was increased. Dirichlet boundary conditions were only applied to the outside of the surrounding tissue, as well as the distal end of blood vessels. Further, in contrast to [8], the gap function $g_{N}$ of the friction and contactless contact problem between heart and surrounding tissue, used to represent the pericardium, was changed to

$$
\begin{aligned}
g_{N} & =e^{2} \cdot x^{2} \cdot e^{-2 x} \cdot \mathbf{n}^{\mathbf{m}}, \text { with } \\
x & =\frac{d_{F \max }}{\left\|\mathbf{x}^{\mathbf{s}}(\xi)-\mathbf{x}^{\mathbf{m}}(\xi)\right\|},
\end{aligned}
$$

with $\mathbf{n}^{\mathbf{m}}$ being the normal of the master element, $x^{m}$ the projection of the epicardial surface element in normal direction towards the surrounding tissue surface, $x^{s}$ the other way around, and the distance $\left(d_{F \max }\right)$ at which the force is at its maximum $(2 \mathrm{kPa})$ being set to $1 \mathrm{~mm}$.

Pressure within the ventricles was modulated by defining cavities of the left and right ventricle/atria and assuming a uniform pressure on the endocardium within, respectively. 
Based on the computed deformation, stretch is interpolated to the nodes of the electrophysiological mesh for every $\mathrm{ms}$, thereby closing the feedback loop between deformation and electrophysiology. The mechanical model was initialized according to [8], by applying a negative pressure, resetting the stretch, and then inflating the cavities back to their initial volume again.

\subsection{Forward ECG-Calculation}

Based on the resulting mycocyte membrane voltages, the bidomain equations were used to calculate the extracellular potentials within the torso [2]. In order to do so for the dynamic approach, the deforming heart was embedded into a previously determined cavity within the torso. This cavity was the outer fixated surface of the surrounding tissue used in the mechanical part of the simulation. As normally there is no structural connection between epicard and surrounding tissue, the surrounding tissue had to be remeshed for every time step, creating a link between torso and epicard. For the static approach the initial configuration of the deformation was used for the complete ECG, see Fig. 2. Further, to assess the effect of the relationship between repolarization and diastolic phase, the calculated $V_{m}$ were shifted artificially in time. Meaning, that the resulting $V_{m}$ were shifted after calculating the deformation, but before calculating the ECG. Implementing a similar effect by varying channel properties and thus shorting or prolonging action potentials, would most likely lead to a different mechanical response. Thus, this way we were able to separate to what extend the T-wave is influenced by the different phases of the same contraction cycle.

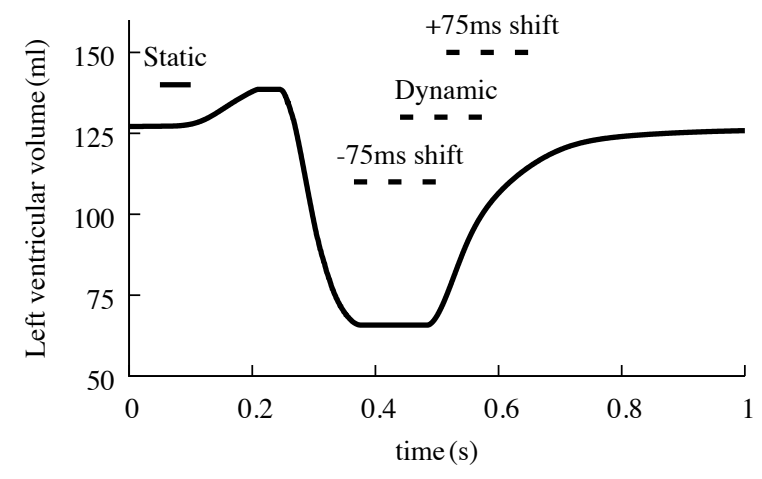

Figure 2. Left ventricular simulated ejection and the respective alignments of the repolarization of the ventricles from the three different setups shown in dashed lines.

\section{Results}

As seen in Fig. 2 in the normal/dynamic setup the repolarization aligns half and half within the isovolumentric relaxation and the relaxation phase of the heart cycle. The $-75 \mathrm{~ms}$ shifted one lies completely in the isovolumetric and the $+75 \mathrm{~ms}$ shifted one in the relaxation phase. An even further positiv shift to the end of the diastole would lead to the same results as the static one.

Fig. 3 shows the resulting ECG in the Einthoven II lead for the different setups and Table 1 the resulting changes in T-wave properties. In a direct comparison, the ECG calculated using the dynamic approach showed an increase in amplitude of $8 \%$, compared to the static approach. Further, the peak of the T-wave shifted by $\sim 7 \mathrm{~ms}$ towards longer durations. Depending on the calculation of the QT-interval one could argue that thus the QT-interval increased from 308 to $315 \mathrm{~ms}$. Yet, when looking at the T-wave it can be seen that the T-wave itself is not shifted, but rather the peak within the T-wave, making it lean somewhat to the right. By artificially shifting the electrophysiological part of the simulation this effect could be amplified or almost reverted. The most prominent effect can be seen for the $-75 \mathrm{~ms}$ shifted setup, resulting in a further increase in Twave amplitude by $10 \%$ and a further delay in time to peak by $2 \mathrm{~ms}$. In contrast the effects are already almost reverted for the $+75 \mathrm{~ms}$ setup, as the heart is close to being back in its end-diastolic state.

Table 1. Resulting T-wave amplitudes and time between the peaks of Q and T-wave in Einthoven II lead (shifts are deducted).

\begin{tabular}{lcc}
\hline \hline & Amplitude $(m V)$ & TTP $(m s)$ \\
\hline Static & 1.21 & 308 \\
Dynamic & 1.31 & 315 \\
$+75 m s$ shift & 1.22 & 312 \\
$-75 m s$ shift & 1.47 & 318 \\
\hline \hline
\end{tabular}

\section{Discussion}

The model itself has some areas needing improvement. For now, stretch dependent changes in channel properties as well as changes in conduction velocity via deformation of the myocardium were neglected. These could potentially widen the spectrum of impact of deformation on the T-wave but also could affect the QRS-complex and maybe even the P-wave. Further, so far only the Einthoven leads were investigated, a closer look at the Wilson leads might reveal further information. Additionally, in order to make a generalization of the deformational effect, different orientations of the heart within the torso have to be considered. The changes in amplitude of the T-wave presented here are different to the ones from [2], but in agreement with [3]. In [2], a decrease in T-wave amplitude of up to $40 \%$ was shown, independent on the time of repolarization. Whereas in [3] a similar, moderate increase in amplitude 

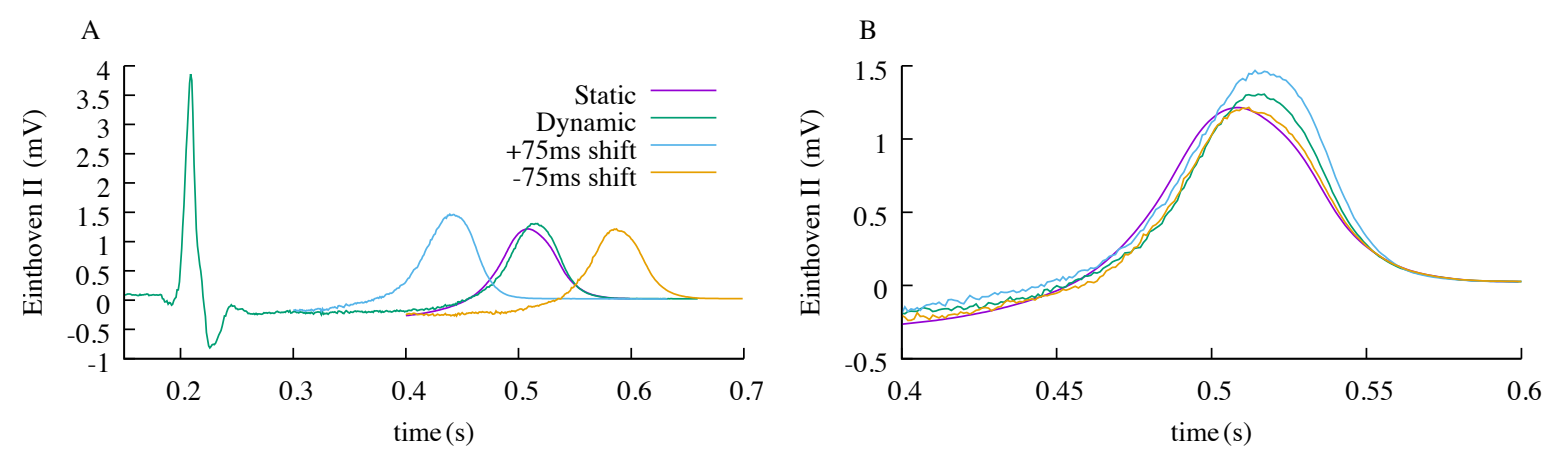

Figure 3. A: resulting Einthoven II lead ECG based on the static, dynamic and the two setups where the resulting membrane voltages were artificially shifted by $75 \mathrm{~ms}$, respectively. B: aligned T-waves, with an increase in amplitude for the dynamic and the positive shifted setup, as well as shift in time to T-wave peak for all the setups, compared to the static one.

was observed.

In conclusion this study shows that the effect of deformation on T-wave amplitude may be regarded small. The potential to affect the morphology of the T-wave, by shifting its respective peak rightwards should be investigated further. Depending on how the QT-interval is defined, this could be seen as a lengthening by up to $10 \mathrm{~ms}$. This could become more important when looking at pathologies such as short QT syndrome as boundaries in diagnostic are often somewhat arbitrary hard values.

\section{Acknowledgements}

The authors Robin Moss and Eike M Wülfers would like to gratefully acknowledge the financial support of the ERC Advanced Grant CardioNECT.

\section{References}

[1] Rice JJ, Wang F, Bers DM, de Tombe PP. Approximate model of cooperative activation and crossbridge cycling in cardiac muscle using ordinary differential equations. Biophysical journal 2008;95(5):2368-90.

[2] Keller DU, Jarrousse O, Fritz T, Ley S, Dössel O, Seemann G. Impact of physiological ventricular deformation on the morphology of the T-wave: A hybrid, static-dynamic approach. IEEE Transactions on Biomedical Engineering 2011;58(7):2109-2119.

[3] Oliveira B, M Rocha B, Barra LP, Toledo E, Sundnes J, Weber Dos Santos R. Effects of deformation on transmural dispersion of repolarization using in silico models of human left ventricular wedge. International Journal for Numerical Methods in Biomedical Engineering 2013;29.

[4] Bayer JD, Blake RC, Plank G, Trayanova NA. A novel rulebased algorithm for assigning myocardial fiber orientation to computational heart models. Annals of Biomedical Engineering 2012;40(10):2243-2254.

[5] Krueger MW, Schmidt V, Tobón C, Weber FM, Lorenz
C, Keller DU, Barschdorf H, Burdumy M, Neher P, Plank G, Rhode K, Seemann G, Sanchez-Quintana D, Saiz J, Razavi R, Dössel O. Modeling atrial fiber orientation in patient-specific geometries: A semi-automatic rule-based approach. Lecture Notes in Computer Science including subseries Lecture Notes in Artificial Intelligence and Lecture Notes in Bioinformatics 2011;6666 LNCS:223-232.

[6] Tusscher KHWJ, Noble D, Noble PJ, Panfilov AV. A model for human ventricular tissue 2010;1522-1539.

[7] Keller DUJ, Weiss DL, Dossel O, Seemann G. Influence of IKs Heterogeneities on the Genesis of the T-wave: A Computational Evaluation. IEEE Trans Biomed Eng 2012; 59(2):311-322.

[8] Fritz T, Wieners C, Seemann G, Steen H, Dössel O. Simulation of the contraction of the ventricles in a human heart model including atria and pericardium: Finite element analysis of a frictionless contact problem. Biomechanics and Modeling in Mechanobiology 2014;13(3):627-641.

[9] Guccione JM, Costa KD, McCulloch aD. Finite element stress analysis of left ventricular mechanics in the beating dog heart. Journal of Biomechanics 1995;28(10):11671177.

[10] Nasopoulou A, Shetty A, Lee J, Nordsletten D, Rinaldi CA, Lamata P, Niederer S. Improved identifiability of myocardial material parameters by an energy-based cost function. Biomechanics and Modeling in Mechanobiology 2017;16(3):971-988.

[11] Balay S, Buschelman K, Eijkhout V, Gropp WD, Kaushik D, Knepley MG, McInnes LC, Smith BF, Zhang H. PETSc Users Manual. Technical report, 2004.

Address for correspondence:

Robin Moss

Institute for Experimental Cardiovascular Medicine

Elsässerstr. 2Q, 79110 Freiburg, Germany

robin.moss@universitaets-herzzentrum.de 